Agency): gastro-duodenal ulcers (including NSAIDs and steroidrelated ulcers), reflux oesophagitis, Zollinger-Ellison's syndrome, and Helicobacter pylori eradication. Inclusion criteria: patients $>65$ years old on at least four home medicines and an anti-ulcer prescription in the ER. Pharmaceutical interventions were recorded and their degree of acceptance calculated. The cost resulting from drug misuse was calculated considering a mean stay in the unit of one day.

Results 111 patients, $70.2 \%$ male, median age 78.9 years-old [65-94]. 94.6\% of patients (92.9\% PPI, 1.7\% H2 antagonists) received one of these agents upon presentation $(95.5 \%$ of them were prescribed de novo), with intravenous pantoprazole the agent mainly involved ( $82 \%$ of cases). $29.7 \%$ of prescriptions did not meet the indications, while this percentage decreased to $12.5 \%$ upon ward admission. The pharmaceutical interventions were accepted in $16.2 \%$ of cases. Monthly, the estimated cost of the off-label use was $€ 1850$.

Conclusions Gastro-protection in the ER did not meet the criteria in nearly $1 / 3$ of patients. This contrasted with the poor acceptance of the pharmaceutical recommendations of discontinuation. The rationale might be the so-perceived harmless profile of these drugs with the short-term use. The rate of off-label prescriptions dropped to half upon ward admission, likely due to thorough revision by the prescriber. Since only patients at a higher risk of suffering from a medicines-related problem were included, the cost resulting from the misuse of anti-ulcer drugs was probably underestimated. In conclusion, forthcoming pharmacy policies should focus on improving the adherence to the indications of both widely-used and expensive drugs, given their financial and health-care impact.

No conflict of interest.

\section{GRP-077 GENERIC MYCOPHENOLATE MOFETIL IN HEART TRANSPLANT RECIPIENTS: IMPLEMENTATION OF ACTIVE PHARMACOVIGILANCE}

doi:10.1136/ejhpharm-2013-000276.077

'L Calixto, ${ }^{1 T}$ Laranjeira, 'A Mirco, ${ }^{2} \mathrm{MJ}$ Rebocho, ${ }^{1} \mathrm{~F}$ Falcão. ${ }^{1} \mathrm{CHLO}$ EPE - Hospital Santa Cruz, Hospital Pharmacy, Lisbon, Portugal; '² CHLO EPE - Hospital Santa Cruz, Transplant Heart Unit, Lisbon, Portugal

Background Immunossupressant drugs have an important role in the prophylaxis of transplant rejection, so they are considered 'critical dose drugs'. Use of a generic immunosuppressant represents a significant cost savings to the medical system. Since safety data for new medicines are always limited, post-marketing surveillance is essential to determine medicines' safety in real life use. With the introduction of generic mycophenolate mofetil (MMF) in CHLO, EPE-HSC, the pharmaceutical services (PHS) have implemented an MMF active pharmacovigilance programme (APP) for HT recipients.

Purpose To describe and quantify suspected adverse drug reactions (ADRs) identified with an APP implemented by the PHS.

Materials and Methods Between 11/2011 and 09/2012, all adult HT recipients who switched from innovator to the generic MMF were included in the MMF APP. This substitution was made under medical supervision and the pharmacist provided the patients with all necessary explanations. Subsequent pharmaceutical assessment was done with a questionnaire (in person or telephone), which identified demographic data, concomitant treatment and suspected ADRs.

Results 55 patients were included in the MMF APP, 78\% male, average age $55 \pm 13[22-76]$ years. 14 patients $(25 \%)$ reported ADRs at MMF switch. These patients had not experienced $\mathrm{ADR}$ s with the innovator drug. The most common $\mathrm{ADR}$ identified were diarrhoea (25\%), stomach ache (12.5\%) and asthenia (12.5\%). All ADRs notifications were reported to the Portuguese National Pharmacovigilance Unit.
Conclusions Most suspected ADRs identified corresponded to MMF's profile ADRs described in the summary of product characteristics. The switch to generic from innovator drug should have a surveillance strategy that includes medical monitoring, patient education and the contribution of all health professionals involved in the patient immunosuppressant regimen in order to create a system that allows adverse reactions to be detected, with the ultimate goal of maximising benefit and minimising risk by promoting safer use of medicines.

No conflict of interest.

\section{GRP-078 GUIDELINE FOR ALBUMIN USE: EFFECT ON COST SAVING}

doi:10.1136/ejhpharm-2013-000276.078

N Yurrebaso Eguilior, J González García, F Gutierrez Nicolas, S Duque Fernández de Vega, S González Ponsjoan, GJ Nazco Casariego. Hospital Universitario de Canarias, Pharmacy, La Laguna, Spain

Background Albumin has been widely used in clinical practise. While some of these indications are supported by the results of randomised studies, others are based only on clinical experience and have not been proved in prospective studies. Efforts should be made to define the indications for albumin use, so that patients gain the maximum benefit from its administration.

Purpose To evaluate the cost saving obtained by the implementation of a guideline for albumin use in a 737-bed hospital.

Materials and Methods Retrospective study that compared albumin use in two periods: July-September 2012 vs. July-September 2011. In June 2012 the guideline for albumin use was distributed to the medical staff. Physicians were requested to complete a form for each albumin order indicating the type and amount of albumin, the clinical service, and the indication for use. Albumin use data and costs were obtained from pharmacy service management system $\left(\mathrm{SAP}^{\circledR}\right)$ and were tabulated using the Exce ${ }^{\circledR}$ software.

Results The total amount of albumin ordered during the study period was $29.360 \mathrm{~g}(€ 63,246)$ vs. $53.195 \mathrm{~g}(€ 108,617)$ for the same period during 2011, which means a reduction of $45 \%$. In terms of cost, the saving obtained amounted to $€ 45,371$ (58\%). The albumin use by specialty had also changed; a major decrease in use of albumin was observed for Anaesthesiology 4,000 g (75\%), General Surgery 3,080 g (65\%), Nephrology 4,900 g (64\%), Internal Medicine $3,860 \mathrm{~g}(56 \%)$, Haematology 1,410 g (53\%) and Digestive 1,400 g $(30 \%)$. On the other hand, Haemodialysis significantly increased its use of albumin to $2,805 \mathrm{~g}$ (65\%), although within the approved indication of plasmapheresis.

Conclusions An albumin use guideline with restrictions focused on albumin prescriptions had suficient efficacy to reduce consumption and save cost. In our hospital guideline the cost of implementation decreased a 58\% (€181,484 per year).

No conflict of interest.

\section{GRP-079 GUIDELINES FOR CHEMOTHERAPY EXTRAVASATION}

doi:10.1136/ejhpharm-2013-000276.079

${ }^{1} \mathrm{M}$ Morgado, ${ }^{2} \mathrm{M}$ Mendes, ${ }^{1} \mathrm{R}$ Oliveira, ${ }^{\prime} \mathrm{S}$ Morgado. 'Hospital Centre of Cova da Beira, Pharmaceutical Services, Covilhã, Portugal; ${ }^{2}$ University of Beira Interior, Health Sciences Faculty, Covilhã, Portugal

Background The administration of intravenous cytotoxic drugs plays a key role in cancer treatment and due to the overall increase in intravenous chemotherapy there has been an increasing incidence of chemotherapy extravasation. Therefore, it is advisable to have updated guidelines that direct the treatment of intravenous cytotoxics extravasation.

Purpose To develop guidelines for the treatment of cytotoxic extravasation, which contained the management algorithms, 
antidotes and treatments that should be performed, as well as risk factors and strategies to prevent extravasation.

Materials and Methods A literature review was performed, through research and analysis of guidelines and articles obtained from PubMed since January/2000 to September/2012, intersecting the terms 'cytotoxic extravasation', 'chemotherapy extravasation' and 'extravasation treatment'. The summary of product characteristics of all of intravenous cytotoxics available in Portugal was also reviewed. Some holders of market authorization were also contacted whenever we considered additional information was required.

Results A total of 42 intravenous antineoplastics available in Portugal were analysed, distributed as follows based on tissue injury after extravasation: 16 vesicant products, 16 irritants and 10 neutral products. A summary table was created with the risk factors (e.g., vesicant drugs, higher drug concentrations, previous vinca alkaloids, elderly, impaired sensory perception, generalised vascular disease) and measures that prevent extravasation (e.g. ensure that the IV site can be clearly visualised, do not use a butterfly needle with a vesicant drug). Nine individual algorithms were developed, according to the latest guidelines, which guide the work of healthcare professionals in case of extravasation (e.g., measures for immediate treatment, applying heat/cold, recommended antidote and instructions for its use). A list was drawn up with all cytotoxics, each being identified with a colour, which corresponded to the colour of the separator with the algorithm to treat its extravasation. An extravasation kit was also designed and a model for document the appropriate recording of extravasation and clinical monitoring of the patient. Conclusions The guidelines developed are a valuable tool for all hospital services that prepare and administer injectable chemotherapy, contributing to responding quickly and effectively to episodes of extravasation.

No conflict of interest.

\section{GRP-080 HAEMATOLOGICAL TOXICITY SECONDARY TO TREATMENT WITH DIAZOXIDE: A CASE REPORT}

doi:10.1136/ejhpharm-2013-000276.080

MD Alonso Castañé, A Martin Sanz, C Guindel Jimenez, J Ortiz de Urbina, I Yáñez González, M Sáez Villafañe. Complejo Asistencial Universitario de León, Farmacia, León, Spain

Background Hyperinsulinism is a rare cause of persistent hypoglycaemia in the neonatal period. Tolerance of diazoxide is usually excellent.

Purpose To describe a case of normocytic anaemia secondary to treatment with diazoxide in an infant diagnosed with congenital hyperinsulinism.

Materials and Methods A retrospective review of medical records from admission in February 2012 to the current situation and a PubMed search of possible cases of this adverse effect.

The patient was a 17-day-old infant who was treated with diazoxide (maximum dose $25 \mathrm{mg} / \mathrm{kg} /$ day) with good response, allowing the progressive discontinuation of the IV glucose and glycaemia control. The patient was started at a dose of $45 \mathrm{mg} /$ day which has been increased to the current dose of $140 \mathrm{mg} /$ day to encourage weight gain.

Results Normocytic and normochromic anaemia gradually because established with tachycardia (decrease in $\mathrm{Hb}$ from $14 \mathrm{mg} / \mathrm{dl}$ to $8.7 \mathrm{mg} / \mathrm{dL}$ in 15 days), which was thought to be associated with diazoxide, as the other parameters were within normal ranges (echocardiography, thyroid function, iron deficiency study).

The haematological toxicity of diazoxide to be usually thought to be neutropenia and thrombocytopenia although anaemia is rarely described. This reaction was notified by the yellow card scheme to the regional pharmacovigilance system. An evolutionary anaemia study was carried out and monitored by transfusions of packed erythrocytes.

Results Hematologic toxicity is thought to be dose dependent and indicates withdrawal of the drug. After a benefit/risk review, the patient is currently still on diazoxide, although other possible treatment options were raised.

After a search in PubMed, we found a single case of anaemia and febrile neutropenia secondary to treatment with diazoxide in an adolescent with hyperinsulinism which was resolved after withdrawal of the drug. This adverse effect may be considered odd.

The importance of Pharmacy Services and other health professionals in reporting adverse reactions is appreciated for the safe use of drugs.

No conflict of interest.

\section{GRP-081 HEALTH INFORMATION TECHNOLOGY AND STRESSORS HOW TO MEASURE AND ELIMINATE THEM}

doi:10.1136/ejhpharm-2013-000276.081

${ }^{1} \mathrm{M}$ Fleury, ${ }^{2} \mathrm{~L}$ Bouchoud, ${ }^{1 P}$ Bonnabry. 'Geneva University Hospitals, Pharmacy/School of pharmaceutical sciences/University of Geneva/University of Lausanne, Geneva, Switzerland; ${ }^{2}$ Geneva University Hospitals, Pharmacy, Geneva, Switzerland

Background The daily tasks of hospital personnel are regularly interrupted. Unexpected breaks in work patterns act as supplementary cognitive burdens on health workers (hence the term stressors) and can lead to errors because they break up the logical flow of clinical tasks.

Purpose To examine whether the introduction of Health Information Technology (HIT) (CytoAdmin - a scanning system for matching patients to their chemotherapy treatment protocols) to a cancer outpatient unit had any immediate effect on stressors, with the broader aim of then reducing their types and frequency.

Materials and Methods Based on techniques drawn from the field of Human Factors and Ergonomics (HF\&E) [1], we established a protocol for carrying out ergonomic evaluation and measuring stressors. The System Engineering Initiative for Patient Safety model was our guiding principle [2]. The protocol covered all tasks in the unit and included field observations, listing stressors (number, type) observed during the introduction of the HIT, and suggesting process redesign methodologies.

Results During the first 6 days of CytoAdmin's introduction, we carried out $31 \frac{1}{2}$ hours of observation of stressors and identified 89 different types (2.7 stressors/hour). The HIT itself generated 21 new stressors (24\% of the total). Amongst these were the insufficient number of computers needed to complete tasks, technical hardware problems and the inclusion of scanning in a wellestablished daily care routine. Ergonomic redesign of workflows allowed us to neutralise all new stressors. Other major stressors were telephone calls (13 types of stressors, 15\%), followed by consulting a physician ( 9 types, $10 \%$ ).

Conclusions The introduction of this HIT increased the number of stressors by creating new ones. The HF\&E system developed was efficient at detecting new stressors, redesigning the process and eliminating them. Although these methodologies are timeconsuming, ergonomic evaluations are essential for the satisfactory and safe use of newly-introduced HIT.

\section{References}

1. Carayon P. (Editor) Handbook of Human Factors and Ergonomics in Health Care and Patient Safety, $2^{\text {nd }}$ edition, Lawrence Erlbaum Associates, Hillsdale, NJ, 2012.

2. Carayon P, et al, Work system design for patient safety: The SEIPS model. Qual Saf Health Care 2006;15(1):50-58

No conflict of interest. 